\title{
Determination of Atom-Surface van der Waals Potentials from Transmission-Grating Diffraction Intensities
}

\author{
R. E. Grisenti, W. Schöllkopf, J. P. Toennies \\ Max-Planck-Institut für Strömungsforschung, Bunsenstraße 10, 37073 Göttingen, Germany \\ G. C. Hegerfeldt and T. Köhler \\ Institut für Theoretische Physik, Universität Göttingen, Bunsenstraße 9, 37073 Göttingen, Germany
}

(April 15, 2018)

\begin{abstract}
Molecular beams of rare gas atoms and $\mathrm{D}_{2}$ have been diffracted from $100 \mathrm{~nm}$ period $\mathrm{SiN}_{x}$ transmission gratings. The relative intensities of the diffraction peaks out to the 8 th order depend on the diffracting particle and are interpreted in terms of effective slit widths. These differences have been analyzed by a new theory which accounts for the long-range van der Waals $-C_{3} / l^{3}$ interaction of the particles with the walls of the grating bars. The values of the $C_{3}$ constant for two different gratings are in good agreement and the results exhibit the expected linear dependence on the dipole polarizability.

34.50.Dy, 03.75.Be
\end{abstract}

Already in 1932 Lennard-Jones [1] predicted that the van der Waals interaction of atoms and molecules with solid surfaces is given by

$$
V=-\frac{C_{3}}{l^{3}}, l \gtrsim 10 \AA
$$

where $l$ is the distance from the surface. This potential plays an important role in understanding virtually all static (thermodynamical) and dynamical aspects of gas adsorption phenomena. Despite its importance, very few experimental determinations of $C_{3}$ have so far been reported and most of our present knowledge is based on theoretical estimates [2]. The pioneering experiments by Raskin and Kusch on the deflection of Cs atoms from a conducting metal surface [3] have recently been extended to alkali atoms in high Rydberg states by measuring the transmission through $8 \mathrm{~mm}$ long narrow $(2-9 \mu \mathrm{m})$ channels as a function of their principal quantum number $n$ [4]. Similar techniques have also been applied to the interaction of alkali atoms in their ground state [5]6 or in low excited states [7. Although the scattering of many different atoms and molecules from solid single crystal surfaces has been extensively studied, the reflection coefficients are relatively insensitive to the weak long range attractive forces since the collisions are largely determined by the reflection from the hard repulsive wall close to the surface [8].

Here, a new atom optical technique using transmission grating diffraction [9, 10] of molecular beams is employed. The van der Waals force causes a change in the diffraction intensities just as a smaller slit width would. A newly developed theory makes it possible to interpret measurements over a range of different beam energies in terms of the potential constant $C_{3}$. For an incident plane wave the diffraction peak heights depend on the number of illuminated slits $N$, as $N^{2}$. With $N=100$ slits the gain in sensitivity is about four orders of magnitude over previous experiments.

The measurements were made with a previously described [10] molecular beam diffraction apparatus. The beams are produced by a free jet expansion of the purified gas through a $5 \mu \mathrm{m}$ diameter, $2 \mu \mathrm{m}$ long orifice from a source chamber at a temperature $T_{0}$, into vacuum of about $2 \times 10^{-4} \mathrm{mbar}$. At $T_{0}=300 \mathrm{~K}$ the source pressure $P_{0}$ was 140 bar for $\mathrm{He}, \mathrm{Ne}, \mathrm{Ar}$ and $\mathrm{D}_{2}$ and 50 bar for $\mathrm{Kr}$. At lower source temperature $P_{0}$ was reduced to avoid cluster formation. The atomic beams are characterized by narrow velocity distributions with $\Delta v / v \approx 2.1$ $\%(\mathrm{He}), 5 \%(\mathrm{Ne}), 7.6 \%\left(\mathrm{D}_{2}\right), 7.7 \%(\mathrm{Ar})$, and $10 \%(\mathrm{Kr})$ at $T_{0}=300 \mathrm{~K}$, where $\Delta v$ and $v$ denote the full half width and the mean value, respectively. After passing through the $0.39 \mathrm{~mm}$ diameter skimmer the beam is collimated by two $10 \mu \mathrm{m}$ wide and $5 \mathrm{~mm}$ tall slits $6 \mathrm{~cm}$ and $48 \mathrm{~cm}$ downstream from the source before it impinges on the silicon nitride $\left(\mathrm{SiN}_{x}\right)$ transmission grating with a grating period of $d=100 \mathrm{~nm}$ and $5 \mathrm{~mm}$ high slits with nominal widths of $s_{\text {nom }}=50 \mathrm{~nm}$ [11] placed $2.5 \mathrm{~cm}$ behind the second collimating slit. The diffraction pattern is measured by rotating the electron impact ionization mass spectrometer detector around an axis parallel to the grating slits. A third, $25 \mu \mathrm{m}$ wide slit, $52 \mathrm{~cm}$ downstream from the grating, provides a measured angular resolution of $70 \mu \mathrm{rad}$ (FWHM).

Transmission measurements with $\mathrm{He}$ and $\mathrm{Kr}$ atomic beams indicate that the grating bars have a truncated trapezoidal profile (thickness in the beam direction $t$ ) 12, 13 with the narrow face towards the incident beam. The measured wedge angles $\beta$ and geometrical slit widths $s_{0}$ (see below) are listed in Table 1 .

The diffraction measurements are illustrated in Fig. 1 for four inert gases as a function of the perpendicular wave vector transfer $\kappa=k \sin \vartheta$, where $\vartheta$ is the diffraction angle. The area under the $n$-th order diffraction peak, $I_{n}$, is proportional to the grating slit function evaluated at the diffraction angle of the maximum position, $\vartheta_{n}$. For this grating, I, which has equally wide bars and slits, the zeros of the slit function coincide with the even 
diffraction orders 14], which are therefore expected to vanish. Whereas for He this is almost the case, for the heavier rare gases, an increasing deviation is observed. For example, the small He intensity ratio of the second and third order peaks is slightly larger for $\mathrm{Ne}$, almost unity in the case of Ar and, finally, for $\mathrm{Kr}$ is greater than one. Similar trends are observed for the ratio of the sixth and fifth order peaks and in the ratio of the most intense zeroth and first orders, which increases significantly from about 0.39 for He to about 0.52 for Kr.

These differences are attributed to the interaction of the atoms with the bar walls, Eq. (11), which so far has not been accounted for in the theory of atom/molecule diffraction. For a plane wave $e^{i k z}$ incident on a transmission grating with perfectly reflecting grating bars and with an additional (attractive) potential at the bar sides, the diffracted wave function is, for large $r$,

$$
\psi(\mathbf{r}) \underset{r \rightarrow \infty}{\longrightarrow} f(\vartheta) \frac{e^{i(k r-\pi / 4)}}{\sqrt{r}},
$$

where $r^{2}=x^{2}+z^{2}$ is in the scattering plane normal to the height of the slits. The scattering amplitude $f(\vartheta)$ is determined by the grating transmission function $\psi(x, 0)$, i. e. by the wave function at the far side slit boundaries $(z=0)$, which depends on the attractive potential. Huygens' principle [14] yields

$$
f(\vartheta)=\frac{\cos \vartheta}{\sqrt{\lambda}} \int_{\text {slits }} d x \psi(x, 0) e^{-i k x \sin \vartheta} .
$$

If the slit and the bar widths are much larger than the de Broglie wave length $\lambda$, the intensity $I(\vartheta)=|f(\vartheta)|^{2}$ can be written as a product

$$
I(\vartheta)=\left(\frac{\sin \left(\frac{1}{2} N k d \sin \vartheta\right)}{\sin \left(\frac{1}{2} k d \sin \vartheta\right)}\right)^{2}\left|f_{\text {slit }}(\vartheta)\right|^{2},
$$

where $N$ denotes the number of slits and $\left|f_{\text {slit }}\right|^{2}$ is the slit function. Thus, the atomic diffraction pattern consists of principal maxima at the diffraction angles $\sin \vartheta_{n}=$ $n \lambda / d, n=0, \pm 1, \pm 2, \ldots$ while $\left|f_{\text {slit }}(\vartheta)\right|^{2}$ plays the role of an envelope function. Eq. (3) gives, after a change of variable from $x$ to a variable with the origin at the edge of a slit, $\zeta \equiv s_{0} / 2-x$,

$$
f_{\text {slit }}(\vartheta)=\frac{\cos \vartheta}{\sqrt{\lambda}} 2 \int_{0}^{\frac{s_{0}}{2}} d \zeta \cos \left[\kappa\left(\frac{s_{0}}{2}-\zeta\right)\right] \tau(\zeta),
$$

where $\tau(\zeta)=\psi\left(s_{0} / 2-\zeta, 0\right), 0 \leq \zeta \leq s_{0} / 2$, is the singleslit transmission function.

It is instructive to first deduce the general structural form of $f_{\text {slit }}(\vartheta)$. Since the grating bars reflect those atoms which touch the bar walls, the wave function in the slit vanishes at the walls, i. e. $\tau(0)=0$. Taking this into account and after a partial integration Eq. (5) becomes

$$
f_{\text {slit }}(\vartheta)=\frac{\cos \vartheta}{\sqrt{\lambda}} \tau\left(\frac{s_{0}}{2}\right) \frac{e^{i \kappa \frac{s_{0}}{2}} \Phi(-\kappa)-e^{-i \kappa \frac{s_{0}}{2}} \Phi(\kappa)}{i \kappa},
$$

where

$$
\Phi( \pm \kappa) \equiv \int_{0}^{\frac{s_{0}}{2}} d \zeta e^{ \pm i \kappa \zeta} \frac{\tau^{\prime}(\zeta)}{\tau\left(\frac{s_{0}}{2}\right)},
$$

with $\Phi(0)=1$. The logarithm of $\Phi$ can be expanded as

$$
\log \Phi( \pm \kappa)=\sum_{n=1}^{\infty} \frac{( \pm i \kappa)^{n}}{n !} R_{n}
$$

where the complex $R_{n}$ are known as cumulants [15],

$$
R_{1}=\int_{0}^{\frac{s_{0}}{2}} d \zeta \zeta \frac{\tau^{\prime}(\zeta)}{\tau\left(\frac{s_{0}}{2}\right)}=\frac{s_{0}}{2}-\int_{0}^{\frac{s_{0}}{2}} d \zeta \frac{\tau(\zeta)}{\tau\left(\frac{s_{0}}{2}\right)},
$$

etc.. For the small wave-vector transfer $\kappa$ of interest here, only the first two terms are needed in the series Eq. (8). The single-slit amplitude Eq. (6) then becomes

$$
f_{\text {slit }}(\vartheta)=2 \frac{\cos \vartheta}{\sqrt{\lambda}} \tau\left(\frac{s_{0}}{2}\right) e^{-\frac{\kappa^{2}}{2} R_{2}} \frac{\sin \left[\kappa\left(\frac{s_{0}}{2}-R_{1}\right)\right]}{\kappa} .
$$

For a comparison with experiment the surface roughness of the grating bars must be accounted for. In a first approximation roughness has been included by rigid shifts of the individual bar sides (see also Ref. [16]), which are randomly Gaussian distributed. In the case of a weak surface potential, this results in an additional Debye-Waller like damping factor $\exp \left(-k^{2} \sigma_{0}^{2} \sin ^{2} \vartheta_{n}\right)$ in the intensity ratio of the principal maxima, $I_{n} / I_{0}$, where $\sigma_{0}^{2}$ is the variance of the geometrical slit width [13]. Taking this into account, Eq. (何) with Eq. (10) yields

$$
\frac{I_{n}}{I_{0}}=\frac{e^{-\left(\frac{2 \pi n \sigma}{d}\right)^{2}}}{\left(\frac{\pi n \sqrt{s_{\text {eff }}^{2}+\delta^{2}}}{d}\right)^{2}}\left[\sin ^{2}\left(\frac{\pi n s_{\mathrm{eff}}}{d}\right)+\sinh ^{2}\left(\frac{\pi n \delta}{d}\right)\right]
$$

where $\sigma^{2} \equiv \sigma_{0}^{2}+\operatorname{Re}\left(R_{2}\right), s_{\text {eff }} \equiv s_{0}-2 \operatorname{Re}\left(R_{1}\right)$ and $\delta \equiv$ $2 \operatorname{Im}\left(R_{1}\right)$. The first term in the brackets of Eq. (11) leads to a Kirchhoff-like slit function (see e. g. Ref. [13]) with a Debye-Waller term and an effective reduced slit width $s_{\text {eff }}$, while the second term suppresses the zeros of the Kirchhoff pattern, as can be seen in the insets of Fig. 1.

The effective variance $\sigma^{2}$ as well as $s_{\text {eff }}$ and $\delta$ in Eq. (11) can be calculated for the potential Eq. (1). The standard eikonal approximation 17.96 is used to determine the grating transmission function, given by $\psi(x, 0)=e^{i \varphi(x)}$ in the slits and zero elsewhere. The phase shift reads

$$
\varphi(x)=-\frac{1}{\hbar v} \int d z V(x, z),
$$

where $v=\hbar k / m$ is the particle velocity. Taking the trapezoidal bar profile into account, after some algebra 
the single-slit transmission function becomes

$$
\tau(\zeta)=\exp \left[i \frac{t \cos \beta}{\hbar v} \frac{C_{3}}{\zeta^{3}} \frac{1+\frac{t}{2 \zeta} \tan \beta}{\left(1+\frac{t}{\zeta} \tan \beta\right)^{2}}\right] .
$$

An analysis of Eqs. (13) and (9) reveals that $\operatorname{Re}\left(R_{1}\right)$ and hence $s_{\text {eff }}$ is especially sensitive to the potential.

The effective slit width $s_{\text {eff }}$ as well as $\delta$ and $\sigma$ were determined from the experiment by fitting the relative experimental diffraction intensities $I_{n} / I_{1}$ as depicted in the insets of Fig. 1 to the corresponding ratios determined from Eq. (11). These ratios and not $I_{n} / I_{0}$ are compared with theory since small concentrations of clusters in the beams can falsify the $I_{0}$ intensities. The effective slit widths are plotted versus the particle velocity in Fig. 2 (points) for two different gratings. The difference between the effective slit widths for $T_{0}=300 \mathrm{~K}$ beams and the geometrical slit width $s_{0}$ increases from $1 \mathrm{~nm}(\mathrm{He})$ to more than $6 \mathrm{~nm}$ for $\mathrm{Kr}$ as expected from the increasing interaction strength of the van der Waals potential. With increasing $C_{3}$ the slope of the curves also increases. The solid lines in Fig. 2 2 represent least squares fits of the theoretical expression $s_{\text {eff }}=s_{0}-2 \operatorname{Re}\left(R_{1}\right)$, with $R_{1}$ given by Eqs. (9) and (13), to the experimentally determined effective slit widths, which allow for the determination of $C_{3}$ and $s_{0}$. Since He has the smallest polarizability and measurements over the largest range of velocities were possible they were used to determine the values of $s_{0}$ in Table If for each of the gratings. Identical values for $s_{0}$ were obtained from $\mathrm{D}_{2}$ measurements. This value of $s_{0}$ was then used for $\mathrm{Ne}, \mathrm{Ar}$ and $\mathrm{Kr}$, with $C_{3}$ the only remaining fit parameter, and hence for these systems measurements at various velocities are not necessary.

The $C_{3}$ parameters are plotted versus the static electric dipole polarizabilities $\alpha$ in Fig. 3 . The error bars were determined by assuming a realistic uncertainty in the bar geometry by varying $\beta$ by $\pm 2^{\circ}$ in Eq. (13). This uncertainty seems to be the only systematic source of error in the present $C_{3}$ determination and leads to errors of about $20 \%$. Figure 2 indicates that the influence of the surface potential is restricted to distances much smaller than the slit width and therefore, by Ref. [18], corrections due to the finite bar width should be negligible.

Within the error bars the data from both gratings fall on a straight line in agreement with Hoinkes' empirical rule [8]. Accordingly the slope provides information on the optical dielectric constant of the grating material. An approximation to the theoretical expression for $C_{3}$ [19] predicts that $\mathrm{D}_{2}$ should in fact have a slightly smaller ratio of $C_{3} / \alpha$ than the rare gas atoms, while among them $\mathrm{Ne}$ is expected to have the largest ratio. It is satisfying to see that the small deviations from the straight line in Fig. 3 agree with this expected trend.

The big advantage of the present method is its large sensitivity as can be seen from Fig. 2 and its universal- ity. In principle all atoms and molecules are accessible for study. The only restrictions will be to produce gratings of different solids and molecular beams with sufficiently narrow velocity distributions and to reduce the corresponding background in the mass spectrometer detector to assure an adequate signal to noise ratio. The present work also allows for a quantitative understanding of diffraction intensities in atom optics and atom interferometry experiments using transmission structures as optical elements.

We are greatly indebted to Tim Savas and Henry I. Smith of MIT for providing the transmission gratings to us. Further, we thank Dick Manson and G. Schmahl for fruitful discussions.

[1] J. E. Lennard-Jones, Trans. Faraday Soc. 28, 334 (1932).

[2] See e. g. G. Vidali, G. Ihm, H. Y. Kim, and M. W. Cole, Surf. Sci. Reports 12, 133 (1991).

[3] D. Raskin and P. Kusch, Phys. Rev. 179, 712 (1969). See also A. Shih and V. A. Parsegian, Phys. Rev. A 12, 835 (1975).

[4] A. Anderson, S. Haroche, E. A. Hinds, W. Jhe, and D. Meschede, Phys. Rev. A 37, 3594 (1988).

[5] C. I. Sukenik, M. G. Boshier, D. Cho, V. Sandoghdar, and E. A. Hinds, Phys. Rev. Lett. 70, 560 (1993).

[6] A. Landragin, J. Y. Courtois, G. Labeyrie, N. Vansteenkiste, C. I. Westbrook, and A. Aspect, Phys. Rev. Lett. 77, 1464 (1996).

[7] V. Sandoghdar, C. I. Sukenik, E. A. Hinds, and S. Haroche, Phys. Rev. Lett. 68, 3432 (1992).

[8] H. Hoinkes, Rev. Mod. Phys. 52, 933 (1980).

[9] C. R. Ekstrom, D. W. Keith, and D. E. Pritchard, Appl. Phys. B 54, 369 (1992).

[10] W. Schöllkopf and J. P. Toennies, Science 266, 1345 (1994).

[11] T. A. Savas, S. N. Shah, M. L. Schattenburg, J. M. Carter, and H. I. Smith, J. Vac. Sci. Technol. B 13, 2732 (1995).

[12] W. Schöllkopf, J. P. Toennies, T. A. Savas, and H. I. Smith, J. Chem. Phys. 109, 9252 (1998).

[13] R. E. Grisenti, W. Schöllkopf, J. P. Toennies, J. R. Manson, T. A. Savas, and H. I. Smith, submitted to Phys. Rev. A.

[14] A. Sommerfeld, Optics, (Academic Press, New York, 1950).

[15] M. Abramowitz and I. A. Stegun, Handbook of Mathematical Functions, (Dover Publications, New York, 1972).

[16] A. M. Marvin and F. Toigo, Phys. Rev. A 25, 782 (1982).

[17] L. D. Landau, E. M. Lifschitz, Quantum Mechanics (Nonrelativistic theory), Vol. 3, (Pergamon, New York, 1977), pp. 538.

[18] F. Zhou and L. Spruch, Phys. Rev. A 52, 297 (1995).

[19] G. Vidali and M. W. Cole, Surf. Sci. 110, 10 (1981). 
FIG. 1. Diffraction patterns measured with Grating I for $\mathrm{He}, \mathrm{Ne}, \mathrm{Ar}$, and $\mathrm{Kr}$ at the same beam energy $\left(T_{0}=300 \mathrm{~K}\right)$. The insets contain a comparison between least-squares fits of $I_{n} / I_{1}$ determined from Eq. (11) with continuous values of $n$ (solid lines) and Kirchhoff theory (dashed lines) to measured diffraction intensity ratios (points).

FIG. 2. Effective slit widths plotted as a function of the particle velocity for $\mathrm{He}, \mathrm{Ne}, \mathrm{D}_{2}, \mathrm{Ar}$, and $\mathrm{Kr}$ beams. The solid lines are theoretical curves determined from Eqs. (9) and (13) with the $C_{3}$ parameters in Fig. 3. Data points indicate fits of $I_{n} / I_{1}$ determined from Eq. (11) to experimental intensity ratios obtained from diffraction measurements with two gratings.

FIG. 3. Measured $C_{3}$ values of silicon nitride $\left(\mathrm{SiN}_{x}\right)$ obtained in this work plotted as a function of the static electric dipole polarizability of the respective atom, $\alpha$ (see Ref. \&1). The solid line is a linear fit of the data.

TABLE I. Geometrical properties of the three gratings.

\begin{tabular}{|c|c|c|}
\hline \hline grating & $\beta\left[^{\circ}\right]$ & $s_{0}[\mathrm{~nm}]$ \\
\hline I & $7.5 \pm 2$ & 50 \\
II & $8.7 \pm 2$ & $67.5 \pm 0.1$ \\
III & $12.7 \pm 2$ & $71.2 \pm 0.1$ \\
\hline \hline
\end{tabular}




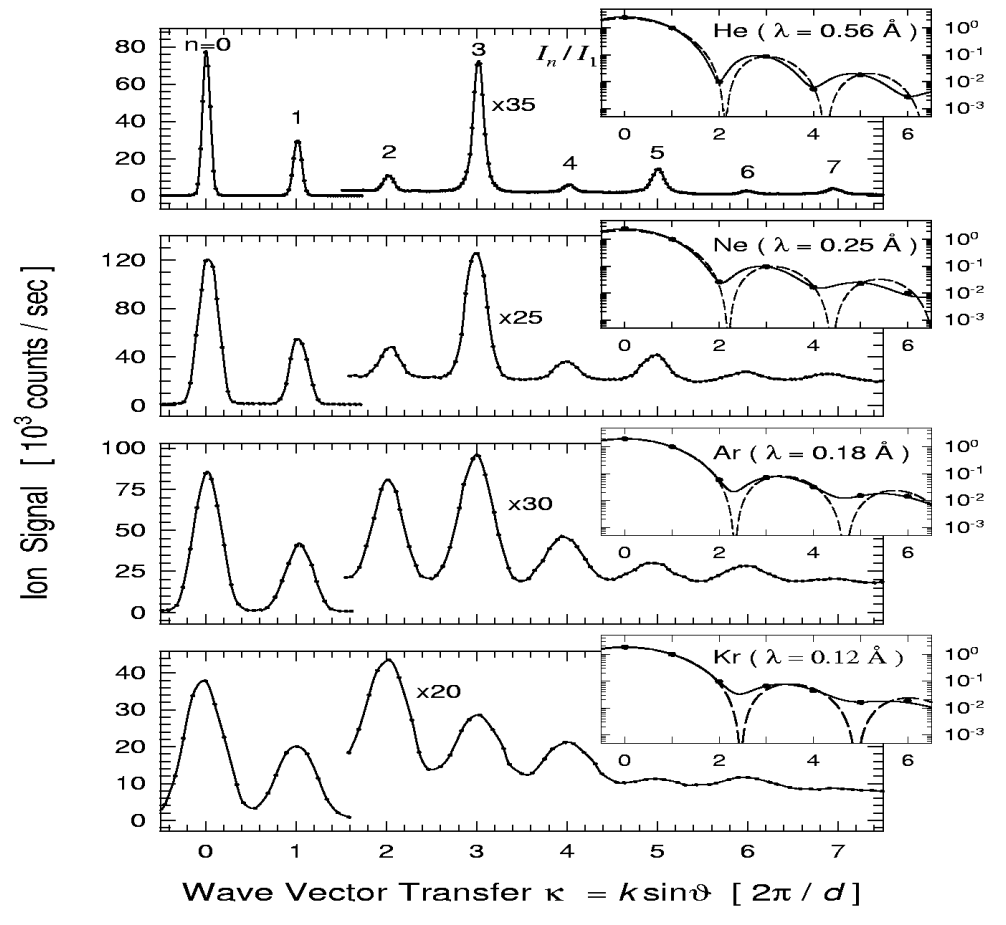




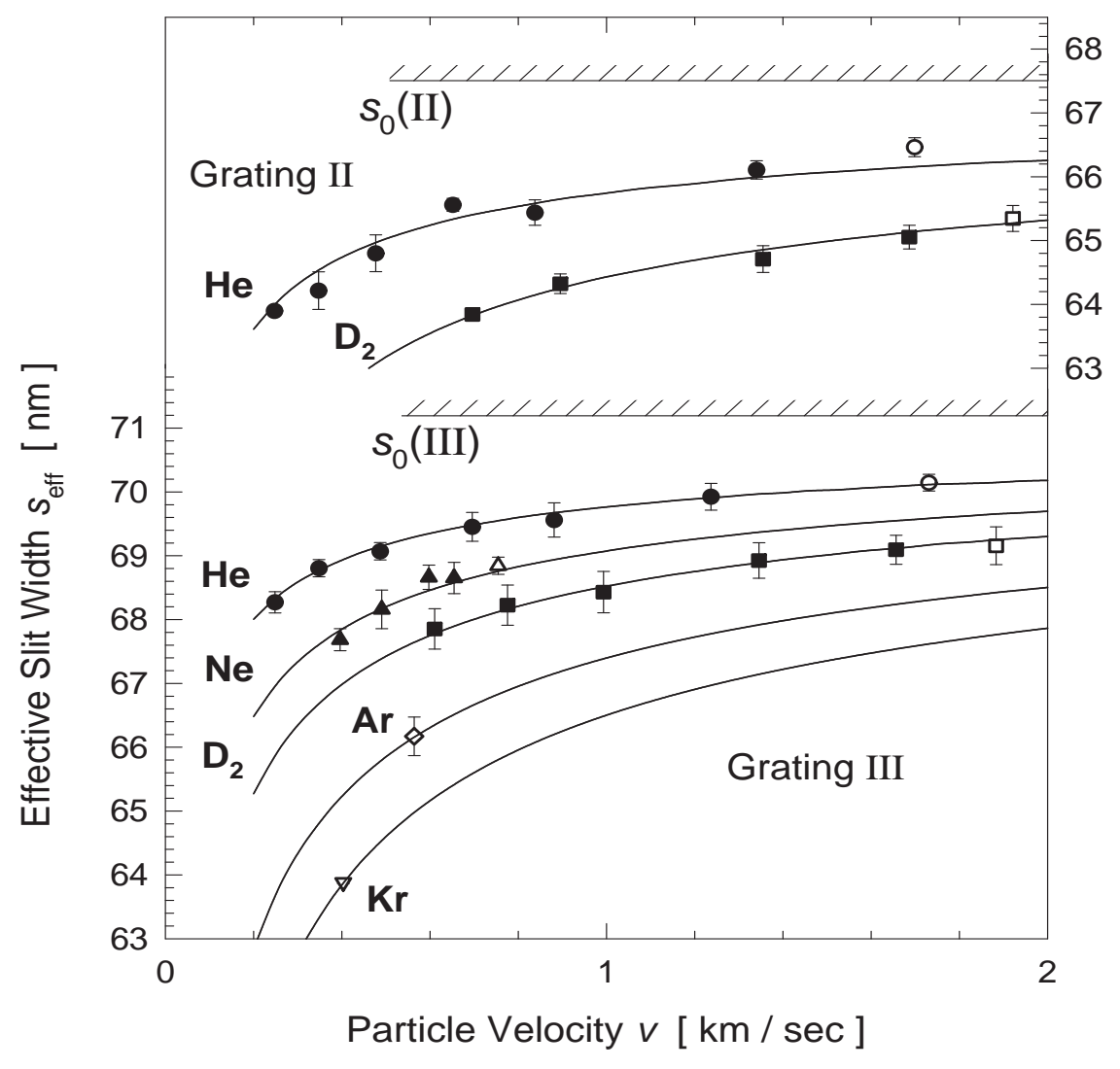




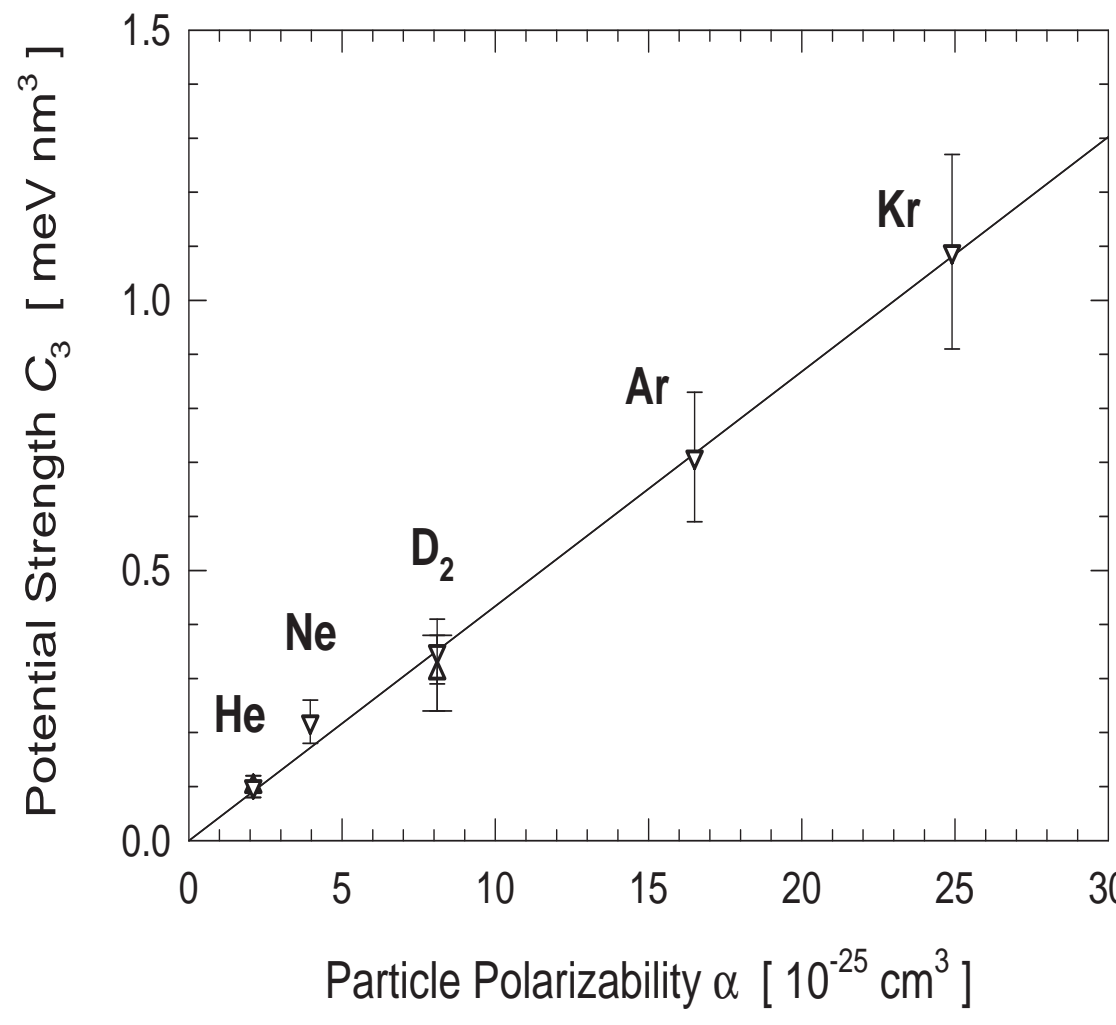

